\title{
Abstracts of the Second Urban Sound Symposium
}

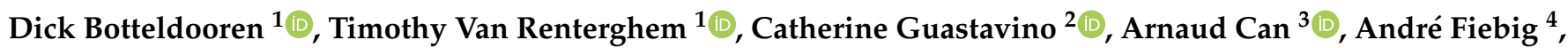

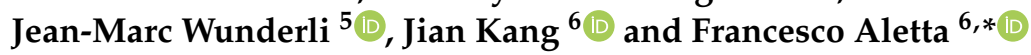

1 Department of Information Technology, Ghent University, 9052 Ghent, Belgium; dick.botteldooren@ugent.be (D.B.); timothy.vanrenterghem@ugent.be (T.V.R.)

2 School of Information Studies, McGill University, Montreal, QC H3A1X1, Canada; catherine.guastavino@mcgill.ca

3 Department of Planning, Mobilities and Environment, Gustave Eiffel University, 44340 Nantes, France; arnaud.can@univ-eiffel.fr

4 Department of Engineering Acoustics, Technical University of Berlin, 10587 Berlin, Germany; andre.fiebig@tu-berlin.de

5 Laboratory for Acoustics and Noise Control, EMPA—Swiss Federal Laboratories for Materials Science and Technology, 8600 Dübendorf, Switzerland; jean-marc.wunderli@empa.ch

6 Institute for Environmental Design and Engineering, University College London, London WC1H 0NN, UK; j.kang@ucl.ac.uk

* Correspondence: f.aletta@ucl.ac.uk; Tel.: +44-(0)-203-108-6597

Citation: Botteldooren, D.; Van

Renterghem, T.; Guastavino, C.; Can,

A.; Fiebig, A.; Wunderli, J.-M.; Kang,

J.; Aletta, F. Abstracts of the Second

Urban Sound Symposium.

Proceedings 2021, 72, 4. https://doi. org/10.3390/proceedings2021072004

Published: 24 May 2021

Publisher's Note: MDPI stays neutral with regard to jurisdictional claims in published maps and institutional affiliations.

Copyright: (c) 2021 by the authors. Licensee MDPI, Basel, Switzerland. This article is an open access article distributed under the terms and conditions of the Creative Commons Attribution (CC BY) license (https:// creativecommons.org/licenses/by/ $4.0 /)$.

\begin{abstract}
Following the successful first Urban Sound Symposium held at Ghent University in 2019, the second edition in 2021 had to face the challenges of the pandemic. The symposium turned this challenge into an opportunity for giving easier access to practitioners and experts from around the globe who are confronted with urban sound in their professional activities. It was organized simultaneously in Ghent, Montreal, Nantes, Zurich, London and Berlin by researchers at Ghent University, Mc Gill University, Université Gustave Eiffel, EMPA, University College London and TU Berlin. The online event created opportunities for interaction between participants at poster-booths, virtual coffee tables, and included social activities.
\end{abstract}

Keywords: urban sound; environmental sounds; soundscape; noise control

\section{Introduction}

The program of the Urban Sound Symposium 2021 included invited keynote talks by eminent researchers from around the world in a single stream as well as in panel discussions on emerging issues. It took place online. The main themes were urban sound planning and design, urban sound propagation and control, sound technologies, and urban soundscape analysis. There was also an option for contributed talks in the form of virtual poster-stands where recent research was showcased and discussed with peers. The latter contributions were subject to review before acceptance. Abstracts of keynote presentations and accepted posters are reported in the following sections. More info on the Symposium can be found on the website (https: / / urban-sound-symposium.org/ (accessed on 20 May 2021)).

\section{Keynote Presentations}

\subsection{Urban Trends and Their Impact on Soundscape}

Dick Botteldooren ${ }^{1}$ and Arnaud Can ${ }^{2}$

${ }^{1}$ Ghent University, Belgium

2 Gustave Eiffel University, France

Urban sound is a product of the way our cities are organized and our society functions. The emergence of new sound sources in the last century has shaped the urban sound environments we know today. Fluidification of living rhythms, sprawl and intensification, 
or the changes in individual mobility practices modify the intensity and temporality of sound sources and thus shape our sonic environment. New areal sound sources may soon appear to complement the light urban freight traffic; mobility provided as a service may cause significant changes in vehicle types and therefore the noise they produce. At the same time, the way urban dwellers perceive and understand the sonic environment is changing. Expectations and sensitivities are changing. Urban sound impacts on city life, its health effects are documented, and it can make a city attractive or not. The living environment in urban areas is gaining more attention as is its resilience against global warming and pandemics. Inequity within a city is accentuated, and urban sound, which closely relates with urban morphology, is one of its causes. But if urban sound is a lively object, so is research and practices that deal with it. New technologies, including smart city and data-rich society-enabled approaches, may also allow policy makers to deploy new modes of governance. This could include urban sound management at a much smaller spatial and temporal granularity.

\subsection{Understanding the Inequity in Risks}

\section{Irene Van Kamp}

National Institute for Public Health and the Environment, The Netherlands

Environmental noise has a negative impact on all of society, but some groups seem more affected than others. While lower socioeconomic status is associated with poorer health in general, it is not clear whether these inequalities arise from increased exposure, increased sensitivity to exposure, increased vulnerability or a combination of those. Socioeconomic status in itself does not cause disease, but apart from biological sensitivity, susceptibility can be a consequence of the exposure itself and thus be related to the places where people live, where they spend their time and their lifestyle. Living in congested building space or in building fabric and street configurations that do not promote healthy behavior can impact on the effects of exposures. Additionally, social risk factors and lack of access to protective measures play an important role. These aspects should be accounted for in health impact assessment by:

1. dividing the study population in relevant subgroups,

2. including info about activity patterns and behaviors,

3. including info about other factors enhancing or reducing susceptibility

4. and by reporting outcomes for subpopulations.

A set of case studies will be presented to illustrate how the well-off often profit more from (environmental) interventions and how (autonomic) demographics selections into highly exposed areas appear to play an important role in these differential effects. Interventions in specifically difficult high-rise old urban towns based on changing the building fabric and building and street configurations could improve these. Driving forces behind these selection mechanisms should be mapped in more detail and should be targeted in policy action. This is aligned with the "health in all policies" approach.

\subsection{Impact of Urban Landscape and Building Configurations on Oppressiveness and Noise} Annoyance in a High-Dense City

\section{Chi-Kwan Chau}

\section{Hong Kong Polytechnic University, Hong Kong}

The connections between sound perception and the visual environment in densely built urban settings have attracted a lot of attention in recent years. An open view is a priced commodity to dwellers in a spatially contested city such as Hong Kong. However, there is very limited understanding about the extent to which views encroached by mountains, heavily trafficked roads, greenery and obstructing buildings at various distances affect the responses of noise annoyance and perceived oppressiveness. The foregoing issues were investigated in two studies. The first study recruited 246 participants 
who were presented with 11 audio-visual stimuli comprising simulated views of different percentages of sea, wooded mountain and trafficked road on a mock-up window panel in a semi-anechoic lab setting. Multivariate analyses of the collected responses showed that views embracing mountains close-by could enhance annoyance. In the second study, simulations in immersive virtual reality of an aging high-rise residential neighborhood with spatial and façade attributes at road traffic noise levels between 55 and $65 \mathrm{dBA}$ were presented to 53 participants who gave annoyance and oppressiveness ratings to 16 scenarios. Findings from multivariate models show that the probability of high noise annoyance responses could be significantly lowered with a larger separation distance, the presence of vertical greening and refurbishment on building façades. In addition, decreasing noise level, increasing separation distance, presence of building spacing, vertical greening and refurbished building façades were likely to reduce oppressive feelings. Implicitly, the model suggested that people with exposure to poorly maintained building facades and blocked views would suffer from high oppressiveness and noise annoyance. These adverse impacts could be moderated if vertical greenery were placed on the facades of surrounding buildings.

\section{and Urban Planning Could Help Reduce Them

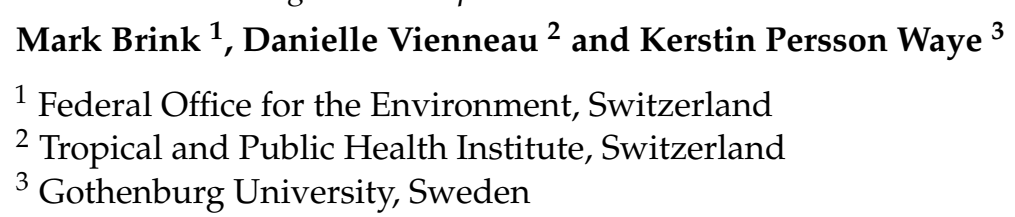

2.4. Noise and the City: Effects of Unwanted Sound on Health and Wellbeing and How Greenery

The health impact of urban noise on annoyance, sleep and cardiovascular outcomes are well established, and there is a growing knowledge on the effects of noise on metabolic diseases, mental health and cognitive functions. Less well researched are how factors in the environment such as greenery can buffer these outcomes, or how urban planning best can lessen the burden. Moreover, we know very little about how noise may affect human health from a life course perspective. This presentation will touch upon these three latter aspects. The life course perspective will be exemplified using sleep as a model. Sleep is vital for physical and mental health, and it is believed to be an important mediator of noise exposure and human health. The life course perspective will touch upon the importance of early exposures and cumulative exposures for the development of sleep patterns and whether this may be of importance for mental and physical health at older ages. Moving outside the bedroom, our local residential setting and broader community environments can also influence mental and physical health. Highlights from large adult cohort studies, investigating the effects of noise and other urban co-exposures on cardiovascular outcomes, will demonstrate the potential benefits of greenery. Availability of neighborhood greenery also came into focus of city planners as a potential means for reducing noise annoyance. Results from a Swiss noise survey revealed that "putting more green into cities" is a promising avenue for lessening the noise burden and the degree to which other measures, e.g., a quiet side on each building, could also help reduce annoyance and sleep disturbances of city dwellers.

\subsection{Public Space Soundscapes-Three Continents, Three Approaches}

\section{Sarah Payne ${ }^{1}$, Hyun In Jo ${ }^{2}$ and Daniel Steele ${ }^{3}$}

${ }^{1}$ Heriot-Watt University, UK

${ }^{2}$ Hanyang University, South Korea

${ }^{3}$ McGill University, Canada

Three speakers in three continents discuss their soundscape research and in particular the methods they have used to investigate public spaces. These methods range from laboratory to in situ and include a wide range of technologies from paper-based questionnaires to immersive virtual reality playback systems. The researchers describe how they utilize 
these methods to document, characterize, and theorize about how people experience public space soundscapes. How these methods lead to practical results for use by professionals and municipalities is also highlighted in their discussions.

\subsection{Smart City Sound Monitoring and Its Applications}

Juan Pablo Bello ${ }^{1}$ and David Bernfeld ${ }^{2}$

${ }^{1}$ New York University, United States

${ }^{2}$ Bruitparif, France

Noise monitoring is a key element of noise pollution mitigation. Innovative sensor networks are being deployed in more and more cities, and it seems important at this point to describe and compare existing innovative approaches. The keynote speakers will present smart city sound monitoring and their applications. The talk will include the presentation of two measurement networks with different characteristics and objectives. The use of machine learning technology, big data analysis and citizen sciences, as well as the aspects of communication with citizens, will be covered. Finally, specific sensors dedicated to particular applications, such as the detection of loud vehicles (acoustic radar) or the monitoring of noise in lively neighborhoods, will also be described. The keynote will conclude with an open discussion on the challenges of noise monitoring in the smart city context.

\subsection{Virtual Reality for Urban Sound}

\section{Catherine Guastavino ${ }^{1}$, Kurt Heutschi ${ }^{2}$ and Julien Maillard ${ }^{3}$}

${ }^{1}$ McGill University, Canada

2 ETH Zurich, Switzerland

${ }^{3}$ Centre Scientifique et Technique du Bâtiment, France

Virtual reality and other immersive technologies are receiving increased attention as ways to support soundscape design in education and practice. This keynote will describe the many ways in which virtual reality can be used by professionals of the built environment, as a training tool to learn about soundscape, as a co-design tool and as a public consultation tool. We will first describe design requirements and then identify the main challenges in creating virtual soundscapes. One challenge relates to the simulation of moving sources (e.g., vehicles) in urban environments. The starting point is an emission signal and a set of impulse responses for discrete source positions from which a continuous source movement is to be simulated. We will share ideas and experiences on the transformation of the original impulse responses into generic ones that allow for simple interpolation strategies. Another challenge is the development of a hybrid method, combining an engineering method and boundary element method, to model complex mitigation options. Audio demonstrations will illustrate the different outcomes.

\subsection{Mapping and Prediction}

\section{Pierre Aumond ${ }^{1}$ and André Fiebig ${ }^{2}$ \\ ${ }^{1}$ Gustave Eiffel University, France \\ 2 Technical University of Berlin, Germany}

Following the environmental noise assessment approach for predicting and mapping noise to identify areas exposed to harmful noises and to derive actions for protecting residents, the newest technological developments promise advanced parameter predictions, including listening. For example, NoiseModelling is a free and open-source tool initially designed to produce environmental noise maps of large urban areas that has been used for the production of sound mapping closer to perception using methods that integrate the multiplicity of sound sources and the dynamics of sound environments. Moreover, sensor networks allowing permanent noise monitoring over larger areas indicate options for instantaneous, dynamic noise maps that are always up-to-date and useable for immediate 
actions. Sensor networks could be used to compute more advanced indicators based on time signals, generating perception-related psychoacoustic noise maps. In this context, the smart city developments call for advanced noise mapping as well as for improved predictions reflecting actual conditions instead of calculations based on some simplified assumptions. However, if sensor networks focus on overall noise, the need for (automatic) source separation immediately arises. The presentation will highlight the current state of mapping and prediction tools and will discuss the progress made and the challenges to overcome.

\subsection{New Approaches to Noise Reduction}

\section{Kirill Horoshenkov ${ }^{1}$, Timothy Van Renterghem ${ }^{2}$ and Jian Kang ${ }^{3}$}

${ }^{1}$ University of Sheffield, UK

2 Ghent University, Belgium

${ }^{3}$ University College London, UK

The first part of this talk, by Horoshenkov, will discuss the ability of living (green) plants to absorb noise. It will be shown that the leaf area density and type of soil substrate are key parameters that affect the ability of these plants to be acoustically efficient absorbers. Different methods of measurement and modelling of the acoustical properties of living plants will be discussed. It will be shown that living plants are also able to scatter a significant proportion of the incident sound energy that is difficult to measure in situ or in a laboratory with standard measurement methods. The second part of this talk, by Van Renterghem, will explore the concept of acoustical landscaping as a natural way to mitigate environmental noise. Raising and depressing the landscape, even to a limited extent, shows to be a valuable but underused approach for reducing sound pressure levels. The focus is on measurement campaigns in public spaces and parks that are heavily exposed to road traffic noise. These results will be positioned in the general framework of urban sound reduction by natural means. The third part of this talk, by Kang, will then explore relationships between sound field and morphology/texture of built-up areas. A number of typical urban areas have been considered, including relatively high-density city built-up areas and low-density town areas, covering a range of building heights, from high-rise to two-story buildings. In terms of sound field, a series of indices have been considered, including spatial noise level Ln. Various sound sources have been taken into account. The work is mainly based on simulation using noise-mapping techniques, as well as Matlab programming, where the effects of building arrangements in a given urban area have also been explored based on genetic algorithms.

\subsection{Urban Planning: Practitioner's Experience with Sound \\ Oscar Breugelmans ${ }^{1}$ and Déborah Delaunay ${ }^{2}$ \\ ${ }^{1}$ National Institute of Public Health, Netherlands \\ 2 Noise and Night-Time Commissioner of Montreal, Canada}

Montreal and Amsterdam are very different cities but face similar challenges to combat environmental noise pollution. In this session, we will explore the major issues that both cities have to deal with and the challenges they face in the future. How do differences between their legislative frameworks and the availability of noise maps and annoyance data shape the actions that can be undertaken? Moreover, what policies, projects and measures have both cities come up with to deal with different noise sources, such as transportation noise, festivals, outdoor terraces and low frequency noise. Amsterdam has a large housing shortage and limited possibilities for building in a region where different functions compete for the remaining space. We will explore results of their noise policy that obliges the construction of a quiet façade. Montreal has great nightlife opportunities but also cohabitation and noise challenges at night. We will examine, in particular, how 
the city develops a night-time policy to permit a diversified, sustainable, healthy and safe nightlife.

\title{
2.11. Participatory Approaches
}

Lisa Lavia $^{1}$, Mattia Cobianchi ${ }^{2}$ and Nadine Schütz ${ }^{3}$

${ }^{1}$ Heriot-Watt University, UK

2 Goldsmiths University of London, UK

${ }^{3}$ Echora, France

The first two talks in this session, by Mattia Cobianchi and Carmen Rosas Pérez, will focus on acoustic inclusivity in the design of indoor and outdoor spaces, promoting the participation of groups with a different sensorial perception. Many guidelines and standards in acoustics have been designed based on average values, often failing to cover the perspective and needs of a large part of the population. This denotes the necessity for rethinking the traditional models and methodologies in favor of broader approaches that include groups such as aural-diverse and neurodivergent individuals and auditory subcultures. The third talk, by Nadine Schütz, will focus on accessibility thinking beyond the regulatory framework and will discuss artistic approaches for a more inclusive and multisensory urban landscape design. Schütz investigates the role of sound in the sensitive relationship between people and their environment, considering the diversity of uses and users in public space, her primary field of intervention. This work also implies considering sound together with the other senses, which participate inseparably in our daily environmental experience. The fourth talk, by Lisa Lavia, will focus on the practical application of ISO soundscape standards in urban planning and development generally, and specifically in the UK. A proposed framework for participatory soundscape planning will be discussed and will focus on the central aspect of soundscape management: assessing the perception of space users (i.e., stakeholders) in context. The necessity of integrating aural diversity and aural accessibility, as quality of life, social justice and equity imperatives in current planning frameworks, will be explored.

\subsection{Designing the Public Space Sound Environment}

\author{
Jordan Lacey ${ }^{1}$, Andy Chung ${ }^{2}$, Gunnar Cerwén ${ }^{3}$ and Trond Maag ${ }^{4}$ \\ ${ }^{1}$ RMIT University, Australia \\ 2 Macau Instituto de Acústica, Macao \\ 3 Swedish University of Agricultural Sciences, Sweden \\ ${ }^{4}$ Urbanidentity-Mobile City Studio, Switzerland
}

This keynote will provide four divergent paths towards a shared outcome: the design of public space environments. Trond Maag will show that working with urban sound can be shaped through the various perspectives of professionals and local communities, supporting the awareness of how surfaces, materials, architecture and landscapes-and how people activate these forms - contribute to the city's sound and identity. Gunnar Cerwén will present a design tool called "Soundscape Actions", consisting of 33 design actions divided into three main categories. Andy Chung will illustrate how virtual technology can be applied to create immersive experiences and to obtain feedback for soundscape design. Jordan Lacey will discuss the electroacoustic practice of transforming motorway parkland soundscapes and methods for integrating spatial sound design with urban furniture.

\section{Poster Presentations}

3.1. "Sons al Balcó": Soundscape of Catalonia during the First COVID-19 Lockdown

Xavier Baño ${ }^{1}$, Pau Bergadà ${ }^{2}$, Alba Egea ${ }^{2}$, Maria Foraster ${ }^{3}$, Marc Freixes ${ }^{2}$, Gerardo José Ginovart-Panisello ${ }^{2}$, Roger Mallol ${ }^{1}$, Xavier Martín ${ }^{4}$, Anna Martínez ${ }^{4}$, Carme Martínez-Suquía ${ }^{2}$, Ferran Orga ${ }^{2}$, Xavier Sevillano ${ }^{2}$ and Rosa Ma Alsina-Pagès ${ }^{2}$

${ }^{1}$ Research and Transference Office, La Salle-Universitat Ramon Llull, Spain 
${ }^{2}$ Grup de Recerca en Tecnologies Mèdia, La Salle-Universitat Ramon Llull, Spain

${ }^{3}$ ISGlobal: Parc de Recerca Biomèdica de Barcelona, Spain

${ }^{4}$ IAR - Integrated Architectural Research, La Salle-Universitat Ramon Llull, Spain

The "Sons al Balcó" project aims to study the effect that the lockdown due to the COVID-19 pandemic has caused on the perception of noise in Catalonia. The research activities co-occurred with a dynamic collaboration of citizens and other stakeholders as a way to create social and environmental impact. The initial scientific hypothesis is that the annoyance coming from outdoor noise, minimized by the lockdown effect, could be associated with better perception of the soundscape by people. The project started with the design of an online test to be conducted by any citizen aiming to contribute for all the territory of Catalonia. A communication campaign was conducted to reach a significant level of participation. During the lockdown, more than 350 questionnaires and videos were collected, and a first map of the soundscape of the confinement in Catalonia was depicted. "Sons al Balcó" is now working to validate this hypothesis in three different ways. The first one, by means of the subjective questionnaires, was conducted with people living in a pre-defined diverse acoustic. The second one combined the measurements conducted by the same citizens answering the questionnaires with the study of the image and sound of all those areas by using the short pieces of video collected by citizens. Finally, the $\mathrm{L}_{\text {Aeq }}$ collected during the lockdown in the cities with operative acoustic sensor networks was studied. "Sons al Balcó" aims to determine the relationship between perception of objective sound levels, characteristics of the urban environment and the landscape and the typology of noise sources.

\subsection{Creative Auralisations for Public Realm Design: A Case Study}

\section{Usue Ruiz Arana}

Newcastle University, UK

This poster presents work-in-progress research that explores the role of creative auralizations in public realm design. The aim of the presentation is to discuss incipient findings and to seek potential collaborators to help develop the research further. The Landscape Institute distinguishes between two types of visualizations: technical visualizations and illustrative visualizations. Technical visualizations are accurate, objective and unbiased and produced as part of Townscape and Visual Impact Assessments or Landscape and Visual Appraisals. They enable relevant authorities to assess the effects of development on the character of an area. Illustrative visualizations are produced for marketing purposes, to showcase the essence of the development or to communicate proposals to stakeholders. Illustrative visualizations are used at different stages of a project for different purposes and form an integral part of the design development of public realm schemes. Through the presentation of a case study, a prospective urban square in Glasgow, this research argues that the development of creative auralizations alongside illustrative visualizations or on their own can help develop a positive soundscape strategy and strengthen the design of the public realm. In the case study, the auralization followed the soundscape assessment of the site and was produced at the concept stage from field recordings of the area and similar environments within Glasgow and the vicinity. The auralization served to explore how sound could be actively used as part of the overall concept for the site, brought soundscape awareness to the wider design team and client and expanded marketing material.

\subsection{Soundscape Design Tools for Professionals}

Richard Yanaky ${ }^{1,2,3}$ and Catherine Guastavino ${ }^{1,2,3}$

${ }^{1}$ School of Information Studies, McGill University, Canada

2 Multimodal Interaction Lab, Canada

${ }^{3}$ Centre for Interdisciplinary Research in Music Media and Technology, McGill University, Canada 
Urban sound planning remains a challenge, as there are still limited options available to help professionals be proactive in their sound design considerations in the early planning stages. This project takes steps towards addressing this need through conducting user research on professionals of the built environment and developing an immersive prototype tool for their urban sound design needs. A soundscape framework is adopted, as this takes a holistic approach by considering not only sounds but also other non-sound-related aspects of the urban space. For example, both the visual surroundings as well as intended activity in the urban space will affect a person's response to a soundscape. In order to present a realistic reproduced soundscape for planning and evaluation, virtual reality is employed. User research has identified several major design considerations, which are being taken into account into this ongoing research and development process. First, since many professionals are unfamiliar with the soundscape approach, a training module is included that gives an overview of foundational soundscape methods. Second, an interactive environment will allow professionals full control to prototype their own urban soundscapes and apply different soundscape intervention techniques. Finally, efforts are placed to integrate this software into a professional's existing software workflow. For example, professionals can import their own 3D model designs, rapidly prototype their own soundscape through the immersive tool and export the soundscape data back to their original software/file. Through these efforts, we hope to help bring further practical proactive soundscape planning methods to professionals.

\subsection{Influence of Green Space Design on Individual Noise Perception \\ Verena Zapf}

Boysen-TU Dresden-Research Training Group, Institute of Landscape Architecture, TU Dresden, Germany

Especially in urban areas, green spaces such as parks are important recreational destinations, yet they are often exposed to high levels of traffic noise. Therefore, the extent to which the design of green spaces affects the perception of noise within the green spaces should be investigated. For this purpose, four soundwalks with a total of 34 participants were conducted in the Großer Garten in Dresden, Germany, in September 2020. A range of acoustic and perceptual properties were measured and examined. Furthermore, the green space was characterized with regard to its vegetative design and the visibility of the noise sources, as well as evaluated by the participants with respect to visual impression and appropriateness. To characterize the soundscape rating, the perceived loudness, pleasantness and eventfulness were asked in the questionnaire. The statistical evaluation has shown that the visual impression, the appropriateness and the masking of the noise sources correlate significantly $(p<0.001)$ with the perceived loudness and pleasantnessbut not with the eventfulness. With regard to vegetation, it was found that the diversity of vegetation correlates significantly $(p<0.001)$ with all three parameters of individual noise perception, whereas the quantity of vegetation does not. Thus, it can be said that appealing design, masking of the noise sources and diversity of vegetation reduce individual noise perception and therefore increase the recreational value.

\subsection{Subjective Responses to Manchester Soundscapes: An Online Experiment}

\section{Maria Luiza de Ulhôa Carvalho ${ }^{1,2}$, William J. Davies ${ }^{1}$ and Bruno Fazenda ${ }^{1}$ \\ ${ }^{1}$ University of Salford, Manchester, UK \\ ${ }^{2}$ Federal University of Goiás, Goiânia, Brazil}

Given the restrictions due to the global pandemic, online listening tests become an alternative way of collecting data. In adaptation, the Manchester Soundscape Experiment Online had a contribution of 158 participants between the months of August to November 2020. The objective was to investigate whether different soundscapes and crowd densities modulate people's subjective responses. Responses were collected on the four soundscape 
perceptual dimensions and on three emotional states (valence, arousal and dominance) using Self-Assessment Manikins. Locations in Greater Manchester were previously suggested to represent the soundscape dimensions as follows: Piccadilly Gardens (vibrant), Peel Park (calm), a bus stop (monotonous), and Market Street (chaotic). A set of 360-degree spatial audio and video recordings were made with three crowd densities (empty, medium and busy) in each location. The audio was down-mixed from first-order ambisonics recordings to head-tracked binaural. With a total of 12 different videos, the samples were separated into two groups (chaotic-vibrant and monotonous-calm) with the three variations of crowd densities. The results demonstrated that the locations and the crowd densities influenced the participants' subjective responses. Eventful, vibrant and arousal semantic differential scales increased with crowd densities in both groups. Locations were rated differently from initial survey indications for the monotonous and chaotic dimensions. The findings suggest that crowd density could be considered as a context factor when evaluating soundscapes.

\subsection{A Soundscape Thematic Guide}

\section{Francesc Daumal i Domènech ${ }^{1}$ and Eduard Minobis López ${ }^{2}$}

${ }^{1}$ Department of Architectural Technology, Polytechnic University of Catalonia, Spain

2 ETSAB Library, Barcelona School of Architecture, Polytechnic University of Catalonia, Spain

We present a new Soundscapes thematic guide, which we have created in the Oriol Bohigas library of the Barcelona School of Architecture, Polytechnic University of Catalonia (http:/ /hdl.handle.net/2117/191568 (accessed on 20 May 2021)). It collects the research that we have been conducting for the topic since the beginning of the 2019-2020 academic year, and which includes books, magazine articles, web articles, academic research, conferences, congresses, regulations, internet resources and sound art, culminating in a terminology. Currently, a sound library (sonotheque) on this topic has also been started. The target audience is acousticians, architects, sound artists, engineers, musicians and urban planners, as well as anyone with general interest in soundscape. The repository has documents from around the world, although it began with the authors' scientific production.

\subsection{Soundscape Recording Techniques for Ecologically Valid Lab Studies}

\section{Hyunkook Lee}

Applied Psychoacoustics Lab, University of Huddersfield, UK

This poster will discuss various recording techniques for lab evaluations of urban soundscape in $360^{\circ} \mathrm{VR}$ reproduction environments. For an ecologically valid urban soundscape evaluation in a lab, it is paramount to make the reproduction environment as realistic and natural as possible. For this, an increasing number of soundscape researchers are using a virtual reality (VR) for a $360^{\circ}$ audio-visual representation. For recording, it is necessary to use a $360^{\circ}$ microphone system that allows for a three-degrees-of-freedom head-tracking, as well as a head-mount display. A dummy head system recommended in ISO12913:2 is not suitable for this purpose as it provides only one spatial perspective. First-Order Ambisonics (FOA) is currently the most accessible and convenient $360^{\circ}$ recording method, but it has several limitations in terms of spatial accuracy, tonal quality, the size of listening area in loudspeaker reproduction and perceived externalization in binaural reproduction. Higher-Order Ambisonics (HOA) is known to provide a higher spatial resolution, but HOA microphone systems tend to be costly, and the loudspeaker playback still produces a suboptimal naturalness and requires a high number of channels for an accurate reproduction. On the other hand, research shows that 8-channel near-coincident 3D microphone arrays such as ESMA-3D and ORTF-3D provide a more natural presentation of a sound field as well as a larger sweet spot in loudspeaker playback environments, which seem to allow for a more ecologically valid lab evaluation of soundscape. This session will present recent 
experimental results comparing different systems in the context of outdoor soundscape, with links to practical audio examples.

\subsection{Sonic Passages: Sound as a Sensory Instrument for Urban Design David Buck ${ }^{1}$ and Eleni-Ira Panourgia ${ }^{2}$ \\ ${ }^{1}$ Department of Landscape Architecture, University of Sheffield, UK \\ ${ }^{2}$ UFR Lettres, Arts Création, Technologies, Laboratoire LISAA, Gustave Eiffel University, France}

This paper discusses a method for examining urban sound environments by proposing alternative sensory readings of the city. While sound has great importance for human health and environmental and social well-being, it is conventionally neglected in favor of functional and visual qualities of space. Sound operates beyond the discontinuity of physical barriers and allows us to perceive activity inside, outside and around sites. Our work brings together landscape research and sound art to suggest new ways for listening to spaces and imagining their auditory future by transforming existing sound sources in terms of spatial quality, location and noise pollution. Techniques of mapping, notation, field recording and sound design are employed to place the focus on sound's direction, diffusion, echo, resonance and dispersion across multiple sites. We argue that transforming soundscapes from different locations at the same time allows us to perceive and manipulate the varied temporalities of urban structures. Our research represents a method to consider as a new approach to sonic urbanism that addresses a silent aporia in the architecture of cities and human experience: the literal and figurative absence of sound.

3.9. Prototyping a Low-Cost Wireless Acoustic Sensor Network with Physical Redundancy to Automatically Classify Acoustic Events in Urban Environments

Ester Vidaña-Vila ${ }^{1}$, Rosa Ma Alsina-Pagès ${ }^{1}$ and Joan Navarro ${ }^{2}$

${ }^{1}$ Grup de Recerca en Tecnologies Mèdia; La Salle-Universitat Ramon Llull, Spain

${ }^{2}$ Grup de Recerca en Internet Technologies \& Storage, La Salle-Universitat Ramon Llull, Spain

Each different noise source in an urban environment has a different impact on citizens' health. Hence, automatically detecting acoustic events in metropolitan areas has arisen as a hot research topic in the last years. Typically, the platforms used to monitor the noise level of cities are composed of expensive sensors placed in specific locations, which limits their coverage area. This work pursues an alternative approach and proposes the use of low-cost sensors (Raspberry Pi Model 2B and USB omnidirectional microphones) deployed in a topology that takes advantage of physical redundancy (i.e., multiple nodes can monitor the same acoustic event simultaneously) to conduct urban sound monitoring. The classification algorithm running on each sensor consists of (1) a deep neural network and (2) a distributed consensus protocol that merges the classification results of neighboring nodes and outputs a final label to the events occurring in real-time. The long-term objective of the work is to physically implement the proposed approach in the Eixample district in the city center of Barcelona to be able to identify different noise sources, which would allow an objective survey of the acoustic comfort of the citizens in the street. Initially, the system was tested using the UrbanSound 8k dataset under laboratory conditions and, in this contribution, it has been validated with data from real operation simultaneous recordings obtained in the city center of Barcelona. Experimental results validate the feasibility of the proposal and justify the usage of physical redundancy to improve the classification accuracy of acoustic events.

\subsection{Melting Buildings: Three Approaches to Sonifying the Arts Tower Illumination}

\section{Lorenzo Prati}

Department of Music, University of Sheffield, UK 
Every night, the four faces of Sheffield's modernist Arts Tower are lit differently as a result of the actions and movement of the students and workers who inhabit the twenty floors of the building. This visual phenomenon is a direct consequence of what Bernard Tschumi refers to as "program"; comprehensive of the events, activities, functions and uses of a building, this is a component inseparable from the space which ontologically defines an architectural structure. The program of a space is an inherently mutable feature, which has been identified by Leatherbarrow as an important factor in defining the notion of architecture's "unscripted performance" (2005). According to this theory, the everchanging patterns of illumination visible on the Arts Tower facades might be considered as part of a larger behavior of the building. If these illumination patterns are observed and gathered as data, sonification can be used effectively to translate this information into a sonic output, enabling new experiences and types of interaction with the urban built environment. Therefore, in this paper I explore how a building can be sonically interacted with and played according to the way in which its façade(s) appears and changes over time, particularly in relation to window illumination and fenestration design. Drawing from the fields of archimusic, ubiquitous music and mobile music studies, three practical approaches are developed and discussed, each one adopting a different bespoke interface to sonify a façade of the Arts Tower, which is used as the case study for all the experiments.

\subsection{Headphones on Trains: A Study on the Use of Headphones on Public Transit}

\section{Chathurthi S. De Silva}

New Jersey Institute of Technology, Newark NJ, United States

The use of headphones is now so commonplace that it is almost second nature for many people to use them. Not only do these people use headphones all the time, but they also use them nearly everywhere, including in urban public spaces. In using headphones, people create their own "private sound environments" in public space. This phenomenon merits attention since the creation of private sound environments may well alter people's experiences of public space. This study answers five research questions about the use of headphones on transit: why people use them, when they begin using headphones and when they discontinue using them, what activities they engage in while using headphones, what they listen to and how using headphones affects their experience. The study was conducted on the PATH train that runs between New Jersey and New York City. Four data collection methods were used: focus groups, in-depth interviews, and online and on-site surveys. Findings indicate that the reasons why people use headphones vary depending on how they use them-whether they play audio or wear them without playing audio. A majority of respondents begin using headphones when they depart from their homes and discontinue using headphones when they reach their homes or places of employment. While using headphones, people engage in various activities, including relaxing and observing surroundings. These activities vary depending on whether people are playing audio or not. Respondents reported that the quality of their experiences on transit improves when they listen to audio and declines when they do not.

\subsection{Sound Fundamentals for Professionals of the Built Environment: A Course for Citymakers}

Edda Bild 1,2, Daniel Steele ${ }^{1,2}$, Richard Yanaky 1,2, Cynthia Tarlao ${ }^{1,2}$, Nicola Di Croce ${ }^{1,2}$ and Catherine Guastavino ${ }^{1,2}$

${ }^{1}$ School of Information Studies, McGill University, Canada

2 Centre for Interdisciplinary Research in Music Media and Technology, McGill University, Canada

Sound researchers have spent at least two decades converging on an established need for professionals of the built environment (PBEs) to exhibit sound "awareness" and "literacy". On the side of practice, PBEs are tasked with integrating and making sense of a wide array of details (of which sound is but one consideration) in order to create 
feasible, coherent and cost-effective plans. To "meet in the middle" and to offer a platform for integrating academic and professional sound-related knowledge, the researchers of Sounds in the City have developed a sound-awareness course designed specifically for PBEs. The course goes beyond often inaccessible theories of sound to instead focus on practical and proven ways that sound can help them achieve their needs, such as how thinking about sound in advance can save money or contribute to urban health goals. This five-session course will cover different facets of the sound experience: (1) Why design with sound in mind? (2) The Making of sound, (3) Perceiving Sound, (4) Understanding Sound and (5) Sound and Society. Each session addresses aspects of policy, design and public health, combining three types of learning: learn by understanding (lectures), learn by seeing/listening (examples) and learn by doing (demonstrations and exercises). Through participating in this course, PBEs will become better equipped to both independently articulate their sound-related needs and work toward explicit sound-related goals, as well as learn when to seek out appropriate outside expertise (e.g., from acousticians, artists or sociologists). A portion of this course will be illustrated through hands-on activities during the symposium.

\subsection{Using Ecoacoustic Indices to Evaluate the Changes of the Urban Acoustic Environment} during the COVID-19 Lockdown in Germany

Jonas L. Fischer 1, Timo Haselhoff 1, Salman Ahmed 1, Bryce T. Lawrence 1, Dietwald Gruehn ${ }^{2}$ and Susanne Moebus ${ }^{1}$

${ }^{1}$ Institute for Urban Public Health, University Hospital Essen, University Duisburg-Essen, Germany

2 Faculty of Spatial Planning, TU Dortmund University, Germany

The lockdown enacted by governments worldwide following the SARS-CoV-2 pandemic caused a substantial decrease in mobility accompanied by anecdotes about a quieting of the city and an increase of natural sounds. We evaluated the change of the urban acoustic environment, comparing SPL and ecoacoustic indices before and during lockdown. We analyzed data from 22 automated sound recording devices located in different land use types in Bochum, Germany. In addition to the $\mathrm{L}_{\mathrm{Aeq}, 24 \mathrm{~h}}$, we explored the normalized difference soundscape (NDSI) and bioacoustic (BIO) indices. BIO measures the total sound activities of biological sources $2-8 \mathrm{kHz}$, while the NDSI quantifies the proportion of anthrophonic to biophonic sounds. We describe the differences of the indices five weeks before and five weeks during the lockdown using 16 March 2020 as reference day. We observed an overall mean noise decrease of $5.1 \mathrm{~dB}(\mathrm{~A})(95 \%$ CI: 5.0,5.2), while the NDSI increased by $0.15(0.14,0.15)$ and BIO decreased by $0.71(-0.6,-0.75)$. We found pronounced peaks in the NDSI and BIO and a high variance before lockdown that diminished markedly during lockdown, however, with distinct differences by land use type and daytime. Our results show an increasing proportion of biological sounds (NDSI) but a decrease of absolute biophonic activity (BIO), likely from anthrophony reduction in the $2-3 \mathrm{kHz}$, suggesting ecoacoustic indices are valuable for describing urban acoustic environment but require parameter fine-tuning for urban areas. Aiming at a transformation to a health-promoting urban mobility, the experience and measurement of high urban sound quality can be a promising approach to mitigating health risks of noise in cities.

\subsection{Towards an Improved Assessment of Noise from Urban Residential Roads}

\section{Rosan Nusselder and Ronald van Loon}

\section{$\mathrm{M}+\mathrm{P}$, The Netherlands}

In the Netherlands, noise from road traffic is the main cause of annoyance experienced in the living environment. In its assessment, as stipulated by law, the health impact of residential roads is underestimated. Roads in historic city centers and urban residential areas differ from larger roads: their axis is typically closer to the building façade, they have a paved road surface and their intensities are low. This has three major consequences. 
Firstly, the Lden is not an accurate indicator because it is based on a continuous stream of vehicles, whereas at short distances from the road residents rather experience single passages. Secondly, paving stones can be over $5 \mathrm{~dB}$ more noisy than asphalt concrete, depending on the material and the geometric pattern, particularly in the lower frequency bands. Moreover, although frequency dependence is incorporated in most calculation methods, what is overlooked is that indoor levels are even higher because of façade characteristics. Even pavements that have lower noise emission than asphalt can lead to higher dBs noise levels inside a building. Lastly, the low vehicle intensities result in low Lden values based on which health effects are predicted to be limited. However, a single truck passage at night is capable of waking a person up from sleep. In conclusion, an improved assessment of noise from urban residential roads is needed. Based on several case studies, we provide recommendations for urban planners and acousticians, including silent paving materials and geometries as well as assessment strategies.

\subsection{Psychoacoustic Parameter Analysis of Urban Green Spaces with Diverse Noise Exposure Conditions}

\section{Omid Samani and Mehmet Ercan Altinsoy}

Institute of Acoustics and Speech Communication, Chair of Acoustic and Haptic Engineering, TU Dresden, Germany

The effects of noise exposure are detrimental to many aspects of life. These effects are exacerbated by the traffic noise in big cities. Urban green spaces can play a vital role in alleviating the complications posed by various types of noise exposure. It obliges urban green spaces to have the required standards for providing residents with a calm and safe environment with low noise exposure. This study aims to investigate the noise exposure situation in urban green spaces. For this purpose, a study was conducted in the grand garden of the city of Dresden. The garden covers 1.8 square kilometers of various landscapes, including water streams, park railways, fountains, bridges, roads for bicycles and pedestrians, etc. Various psychoacoustic parameters are considered in this study. The measurements are carried out in eleven spots with diverse noise exposure conditions, such as areas surrounded by greeneries, areas close to the road, lake and water streams and the areas near paths for pedestrians and cyclists. Eventually, the results provide the status of noise exposure at the measured spots based on psychoacoustic parameters. Analysis of obtained measurements has allowed a conclusion between the spot characteristics and related psychoacoustic parameters to be drawn.

\subsection{Monitoring the Acoustic Environment through a Globally Distributed Network of Sky Hub Trackers}

\section{David Moore, Chris Cogswell and Steve McDaniel}

\section{Skyhub, CA, United States}

Sky Hub is an open-source citizen science project with the goal of studying atmospheric phenomena through observational science. It employs a network of globally distributed "Tracker" nodes built using readily available off-the-shelf hardware. A Tracker combines an Nvidia Jetson-embedded computer, a range of sensors, imaging equipment and sophisticated open-source software to intelligently monitor and identify atmospheric events. A key part of the Sky Hub system going forward will be the incorporation of an acoustic sensor suite to monitor the local soundscape. Data from the sensors will be fused to provide a detailed picture of activity. The goal with the acoustic sensor suite is to provide the capability to cover as wide a frequency range as possible. As such, the team are presently evaluating a geophone and range of Micro-Electro-Mechanical System (MEMs) microphones. The long-term aim is to employ artificial intelligence and machine listening algorithms locally on incoming data to assist in determining atmospheric events. This virtual poster presentation will provide an overview of the Sky Hub system, including the 
aspirations for the acoustic sensor suite. It will also outline potential applications of the data with regard to soundscape monitoring.

3.17. Waterfront Urban Public Space Soundscape Evaluation: Lisbon Case Study from the Users' Perspective

\section{Aline Ventura Nadi}

Instituto Superior Técnico, University of Lisbon, Portugal

Waterfront urban public spaces have their own identity and unique sound characteristics, both derived from a combination of the urban context and water features. Their acoustic assessment is better accomplished by adopting a holistic approach, in which the soundscape appraisal becomes particularly important since it integrates significant factors such as context, user's perception and sound environment. A study of the soundscape of waterfront public spaces from their users' perspective was conducted aiming at understanding their subjective appraisal regarding (1) the relevance attributed to the characteristic sounds of a waterfront environment (water, pier, seagulls, boats), (2) the influence that the visual and the sonic aspects hold on the decision to use these public waterfront spaces and (3) the overall soundscape quality. A questionnaire was administered to 500 users in 10 Tejo River (Lisbon) waterfront urban public spaces between the months of August and December 2020. The results show that the public spaces, where the sound environment most influences their users' decision to use it, have a direct and proportional relationship with its overall soundscape quality and with the degree of importance that is given to the characteristic sounds of waterfront areas. These findings will be correlated with other perceptive factors such as visual features to further understand the factors that contribute to a user's decision to use waterfront public spaces.

\subsection{Exploring Sound Sensing to Improve Quality of Life in Urban Living}

\section{Emily Corrigan-Kavanagh, Mark Plumbley, Marc Green and Andres Fernandez}

University of Surrey, UK

Following the successful application of AI and machine learning technologies to the recognition of speech and images, computer systems can automatically analyze and recognize everyday real-world sound scenes and events. This new technology presents promising potential applications in environmental sensing and urban living. Specifically, urban soundscape analysis could be used to monitor and improve soundscapes experienced for people in towns and cities, helping to identify and formulate strategies for enhancing quality of life through future urban planning and development. Nevertheless, realizing the potential of AI for sound in urban living presents challenges. Current research uses cases that are often unrealistic, lacking appropriate end-user feedback and engagement. In response, this research will employ a range of participatory approaches that will explore how people feel about sounds in their locality and how they would like to change them. The use of participatory approaches will begin with world cafés to direct project outcomes from stakeholders, bringing "AI for Sound" technology out of the lab to realize its potential benefit to society and the economy. A world café is a participatory approach typically set in a café-style environment where participants engage in three 20 min discussions on a question posed in small groups (max of five), ending with a harvest session where everyone together collates conclusions drawn from conversations. In response to the COVID-19 pandemic, we are organizing the world cafés as "virtual world cafés" to engage with residents while adhering to UK national lockdown and social distancing restrictions. Our virtual world cafés follow the original world café style utilizing a video conference tool. For example, virtual Breakout Rooms are used to divide participants into small conversation groups on the same call to help identify themes around how people would choose to change their urban sound environments and the contexts that facilitate this as a way of directing future research into associated AI for sound solutions. This virtual poster reports 
on some of the highlights from these virtual world cafés and outlines plans for forthcoming research.

\subsection{Sounding the City: Tehran's Historical and Contemporary Soundspaces}

\section{Laudan Nooshin}

City, University of London, UK

"Standing on a flat rooftop in north Tehran on a summer's evening, I am immersed in sound: the strains of the call to prayer echoing from local mosques; a rock beat from a passing car; the call of birds circling the mountains; a distant ringtone; the low-level hum of the city below". Tehran, Iran's largest and capital city, is a vibrant metropolis cradled in the foothills of the Alborz mountains and the country's political and cultural center for over 200 years. During this time, it has experienced exponential growth from a small walled town of around 60,000 to a city of more than 8 million. Particularly significant was the period of Pahlavi rule (1925-1979) when an extensive program of urban expansion led to the destruction of historic buildings seen as symbolizing the regressive traditionalism of the preceding Qajar monarchs. The Pahlavis envisioned a capital city fit for a nation that was modern, Western-facing and secular. Official discourses promoted the idea of modernity as incompatible with traditional lifeways and the resulting tensions continue to be felt, seen, and - crucially for this project-heard, in many areas of Iranian life. This poster presentation reports on Sonic Tehran, an interdisciplinary project exploring Tehran as a sounded space and located at the intersection of ethnomusicology, sound studies and urban studies. At the heart of the project is an understanding of sound as an integral part of a rich, multi-sensory urban environment and of listening as an intensely haptic experience. Central questions address the relationship between sound and urban space and the kinds of affective knowledge generated through sonic experiences: How does sound shape, and how is it shaped by, the urban context? What might an in-depth attention to sound reveal about the city, its shared spaces and conflicted history? How are dimensions of difference such as gender, class and religion inflected in the city sounds? And how is sound implicated in the construction of place and community, particularly in the context of rapid urban change and regeneration? Theoretically, the research draws on the work of cultural and urban geographers who argue for an understanding of sound not as a "reflection" of the city, but an active agent in the production of urban space. Case examples to be discussed include a former Qajar prison, now a museum; street performers; and a religious shrine in north Tehran. Tehran offers a significant case study as one of the largest cities in the region, and one that has experienced rapid change and social upheaval in its recent history.

\subsection{Urban Oases, Tranquil Spaces to Relax and Enjoy Nature}

\section{Kaat Smets and Gilke Pée}

Department of Environment and Spatial Development, Flemish Government in Collaboration with the Luwte-Oases Program Team, Belgium

Various Flemish organizations in the domain of environment, spatial planning, health, nature, tourism and culture work together in the "Luwte-oases" program. Luwte-oases are tranquil spaces that function like urban oases for relaxing and enjoying nature. Especially now that living and working are increasingly converging in densely populated urban districts and villages, it is even more important to preserve and create tranquil spaces to establish a healthy environment. This program team focuses on the relationship between a biodiverse, high-quality environment and health. There is a range of spaces that can be called a "luwte-oase", but we focus on working and living environments. They concern green spots that offer tranquility and quietness in a busy environment. The team wants to accelerate the realization of these oases by fully supporting local initiatives. Components discussed include further efforts to raise awareness about the need for tranquil spaces in 
densely urbanized environments, to illustrate the potential of Green Infrastructure with a contribution to health, to valorize the input from early adopters, to provide criteria for qualitative oases and to consider a call to facilitate and support coalition building. Local authorities are a privileged target group, because they also act as a link to other key actors.

3.21. New Developments in the NoiseModelling Tool for Nature-Based Solutions (NBS) Assessment in an Urban Context

\section{Benoit Gauvreau and Nicolas Fortin}

UMRAE, University Gustave Eiffel, France

The aim of this poster is to present new developments in a noise model (and its associated simulation tool), carried out in the framework of the Nature4cities project (EUH2020), in order to assess and quantify the impact of Nature Based Solutions (NBS) on sound environment in an urban context, e.g., horizontal vegetation (low growing plants on ground surface, land use information) and vertical vegetation ("green façades"). This simplified model (regarding other expert models such as PE, TLM, FDTD, etc.) is dedicated to engineering purposes (i.e., impact studies) and is an open-source tool (calculation method + GUI software). It is called NoiseModelling. Thus, this poster presents the basic principles and steps for using NoiseModelling (issued from easily reachable documentation), next introduces a case study (on a district of Nantes city, France) and then gives some examples of results in terms of an acoustic Key Performance Indicator (KPI), i.e., $\mathrm{L}_{\text {den }}$ indicator expressed in global $\mathrm{dB}(\mathrm{A})$ and for some specific third octave bands (e.g., $\mathrm{L}_{\mathrm{den}} @ 500 \mathrm{~Hz}$ and @ $8000 \mathrm{~Hz}$ in this study).

\subsection{Open-Source Modelling Chain for the Dynamic Assessment of Road Traffic Noise Exposure}

\section{Valentin Le Bescond, Arnaud Can and Pascal Gastineau}

\section{Gustave Eiffel University, France}

In light of the growing concern about the adverse effects of noise pollution on health, a better understanding is needed of the relationships between urban transport and individual exposure. To improve the scientific community's modeling capabilities specific to this issue, we are proposing a noise exposure modeling framework that uses agent-based activity, multi-agent travel simulation and a European standardized noise emission and propagation model. Based on two open-source software packages, MATSim and NoiseModelling, this framework aims to simulate the spatiotemporal distributions of daily individual activity and road traffic noise. The proposed approach makes it possible to use all the tools and methods proposed in the NoiseModelling software by importing MATSim outputs, therefore, taking full advantage of the development work carried out within the two communities. As such, it enables both characterizing the individual exposure to road traffic-related noise and investigating noise exposure inequality problems based on the attributes of individuals and their activities.

3.23. A Methodology to Assess the Impact of Driving Noise from Individual Vehicles in an Urban Environment

\section{Sacha Baclet, Siddharth Venkataraman and Romain Rumpler}

The Centre for ECO2 Vehicle Design, The Marcus Wallenberg Laboratory for Sound and Vibration Research, Department of Engineering Mechanics, KTH Royal Institute of Technology, Sweden

Traffic is a major source of environmental noise pollution in urban areas. The present contribution focuses on a methodology designed to assess the impact of the noise generated by individual vehicles on a city's population using NoiseModelling, an open-source library implementing the CNOSSOS-EU model, capable of producing environmental noise maps. The initial step of the proposed method consists of processing microscopic traffic data (simulated in the present contribution), where the traffic intensity is dependent on the time 
of day that is targeted. The micro-traffic data is subsequently used to generate background noise maps by simulating the propagation of traffic noise. Then, the impact of the noise from the vehicle of interest is simulated, based on several parameters (route followed, type of motorization: diesel or hybrid, etc.). Finally, the data are post-processed to calculate the "exceedance" (increase in ambient noise) caused by the vehicle, taking the previously calculated background noise maps as reference. The complete methodology, its underlying assumptions and the associated criteria proposed in order to assess the impact of noise emissions from individual vehicles is demonstrated on a realistic scenario.

\subsection{Designing an Urban Soundscape for People with Dementia: The Correlation between Psychoacoustic Parameter and Human Perception of Safety and Mood}

\section{Arezoo Talebzadeh}

Faculty of Engineering and Architecture, Ghent University, Belgium

One of the soundscape design intentions is creating a comfortable sonic environment by influencing the mood, which involves the appraisal, the restoration, and the improvement of peoples' health and well-being. There is widespread research on the positive effect of soundscape on people with severe or profound intellectual disabilities, such as dementia. Sound also plays a role in generating a feeling of safety and in triggering a specific action; creating sound with this purpose can improve behavior. The WHO estimated that the number of people living with dementia increases by 10 million every year, which shows the importance of considering people with dementia when designing urban environments. This research looks at a selection of sounds that gives persons with dementia a feeling of safety and focuses on their orientation. The urban environment can be confusing and terrifying for people with dementia; using a recognizable soundscape to make the environment feel safer and familiar helps people better navigate the urban area. The research looked at the psychoacoustic parameter of sounds to explore the relationship between these parameters and perception of safety, mood change and response. Hence, the input of a panel of six field experts with different backgrounds was obtained for different sounds. These experts rated on a 5-point scale the degree of suitability of the sound for safety-enhancing, mood-changing or triggering behavior. Further, the research looked at sound saliency concerning safety and mood change, looking at the bottom-up attention mechanism regarding environmental sound because the disease may influence the attention deployment driven by salient stimuli.

\subsection{Pandemic Lockdown Impact on Highway Emissions in Flanders, Belgium}

\section{Luc Dekoninck}

\section{Ghent University, Belgium}

Traffic densities are severely affected by the measures implemented to reduce the transmission of the COVID-19 coronavirus. Home-based work, restrictions on shopping, traveling and eating out and nightly curfews reduced the overall traffic but had the strongest impact on local and regional roads. The traffic on highways was also affected. A significant reduction was recorded for passenger traffic $(-60 \%)$ while the impact on freight traffic was less severe $(-20 \%)$. The Flemish Government has a long-term monitoring location near a major highway in Flanders. In this presentation, the impact of the complex traffic variation at this location is evaluated, including corrections for meteorological variability. These natural experiments provide interesting opportunities to build and test emission models and will enable high quality assessments for more extreme traffic scenarios and traffic interventions in the future. 


\subsection{Noise Labelling of Urban Road Surfaces Using Passenger Cars}

\section{Wout Van Hauwermeiren}

\section{Ghent University, Belgium}

Road surface type but also degradation and wear contribute significantly to rolling noise emissions. As electrical and hybrid vehicles are being introduced, rolling noise remains and becomes a main factor in noise emission for lower-order roads. Monitoring and labelling these roads with regard to urban rolling sound emission requires considerably more effort than monitoring primary roads and highways, due to the sheer total length of these local roads. Therefore, an opportunistic method using vehicles that are on the roads for other purposes is proposed for measuring rolling noise. The proposed method may also have some additional benefits over the standard $\mathrm{CPX}$ regarding the distribution of tires used and the spread of typical driving speeds. However, measurement conditions are uncertain and may influence the results obtained from individual vehicles significantly. Locations that are driven over many times and by multiple cars are used as a reference to eliminate modifiers and confounders. To this end, a machine learning cleaning algorithm inspired by denoising auto-encoders has been designed and implemented.

Author Contributions: F.A. curated the organization of the proceedings. All authors have read and agreed to the published version of the manuscript.

Funding: The Urban Symposium 2021 was sponsored by NTi Audio Solutions for Audio \& Acoustics and HEAD-Genuit-Foundation.

Acknowledgments: The Organizing Committee and the Scientific Committee are grateful to all participants and volunteers of the Urban Sound Symposium 2021 and in particular to the volunteers who supported the development of the online event and side activities: Cleopatra Moshona, Christopher Trudeau, Valentin Le Bescond, Edda Bild, Nicola Di Croce. The Organizing Committee also would like to thank all the panelists who contributed to the panel discussions during the Symposium: Brigitte Schulte-Fortkamp, Francesco Aletta, Juan Bello, Catherine Lavandier, Susanne Moebus, Maryse Lavoie, Andy Chung, Efstathios Margaritis, Trond Maag, Oscar Breugelmans, Judicael Picaut, Trevor Cox, Papatya Nur Dökmeci Yörükoğlu, Christine Kerrigan.

Conflicts of Interest: The authors declare no conflict of interest. 